\title{
Experimental characterization and numerical simulations of the electron source at PITZ
}

\author{
K. Abrahamyan ${ }^{\mathrm{a}}$, J. Bähr ${ }^{\mathrm{a}}$, J.P. Carneiro ${ }^{\mathrm{b}}$, K. Flöttmann ${ }^{\mathrm{b}}$, J.H. Han ${ }^{\mathrm{a}}$, M.v. Hartrott ${ }^{\mathrm{c}}$, \\ M. Krasilnikov a,*, D. Lipka ${ }^{\mathrm{a}}$, V. Miltchev ${ }^{\mathrm{a}}$, A. Oppelt ${ }^{\mathrm{a}}$, B. Petrossyan ${ }^{\mathrm{a}}$, D. Pose ${ }^{\mathrm{a}}$, D. Richter ${ }^{\mathrm{c}}$, \\ S. Schreiber ${ }^{\mathrm{b}}$, L. Staykov ${ }^{\mathrm{a}}$, F. Stephan ${ }^{\mathrm{a}}$ \\ ${ }^{\text {a } D E S Y, ~ Z e u t h e n, ~ G e r m a n y ~}$ \\ ${ }^{\mathrm{b}}$ DESY, Hamburg, Germany \\ ${ }^{\mathrm{c}}$ BESSY GmbH, Berlin, Germany
}

Available online 28 November 2005

\begin{abstract}
The Photo Injector Test facility at DESY Zeuthen (PITZ) works on the optimization of electron sources for Free Electron Lasers (FELs). The main motivation and challenge of PITZ is the production of electron beams with high charge and small normalized transverse emittance. Recent results of electron beam studies at PITZ are presented in this paper. The experimental characterization of the electron source is discussed together with beam dynamics simulations.
\end{abstract}

(C) 2005 Elsevier B.V. All rights reserved.

PACS: 41.75.Ht; 41.85.Ar

Keywords: Photo injector; Emittance

\section{Introduction}

The Photo Injector Test Facility at DESY Zeuthen (PITZ) has been built in order to test and optimize electron sources for Free Electron Lasers (FELs) and future linear colliders. PITZ is a facility that has the purpose to produce intense electron beams with minimum transverse emittance and short bunch length as required for FEL operation. The experimental set-up consists of a 1.5 cell L-band RF gun with a $\mathrm{Cs}_{2} \mathrm{Te}$ photocathode, a solenoid system for space charge compensation, a photocathode laser capable to generate long pulse trains with variable temporal and spatial pulse shape, and an extensive diagnostics section. The slit masks technique is used for the electron beam emittance measurements, the emittance measurements system (EMSY) including single and multislit masks is located at $z=1.62 \mathrm{~m}$ from the cathode. A schematic layout of PITZ is shown in Fig. 1.

\footnotetext{
${ }^{*}$ Corresponding author.

E-mail address: mikhail.krasilnikov@desy.de (M. Krasilnikov).
}

The first stage of the PITZ project has been successfully completed at the end of 2003. A smooth commissioning procedure of the RF gun cavity yielded an operation with up to $900 \mu$ s long RF pulses at $10 \mathrm{~Hz}$ repetition rate and an accelerating gradient at the cathode of about $42 \mathrm{MV} / \mathrm{m}$ [1]. That corresponds to a maximum average power of $27 \mathrm{~kW}$ in the gun cavity with $0.9 \%$ duty cycle. This long RF pulse operation fulfills the TTF2 requirements. The startup conditions of TTF2 on normalized projected beam emittance [2] have been fulfilled. By optimization of the photocathode laser properties together with the RF field and solenoid parameters a minimum normalized projected emittance of $1.7 \pi \mathrm{mm}$ mrad has been achieved for a $1 \mathrm{nC}$ electron beam at a mean longitudinal momentum of $4.7 \mathrm{MeV} / c$.

\section{Photocathode laser}

One of the key issues of a photo injector is the photocathode laser. The laser temporal and spatial profiles have a significant impact on the electron beam performance. In 


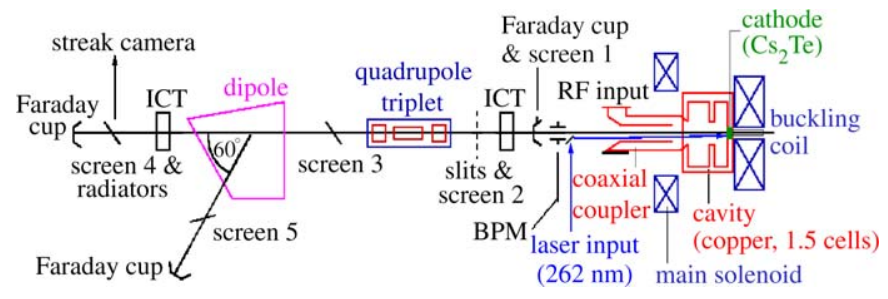

Fig. 1. Layout of PITZ.
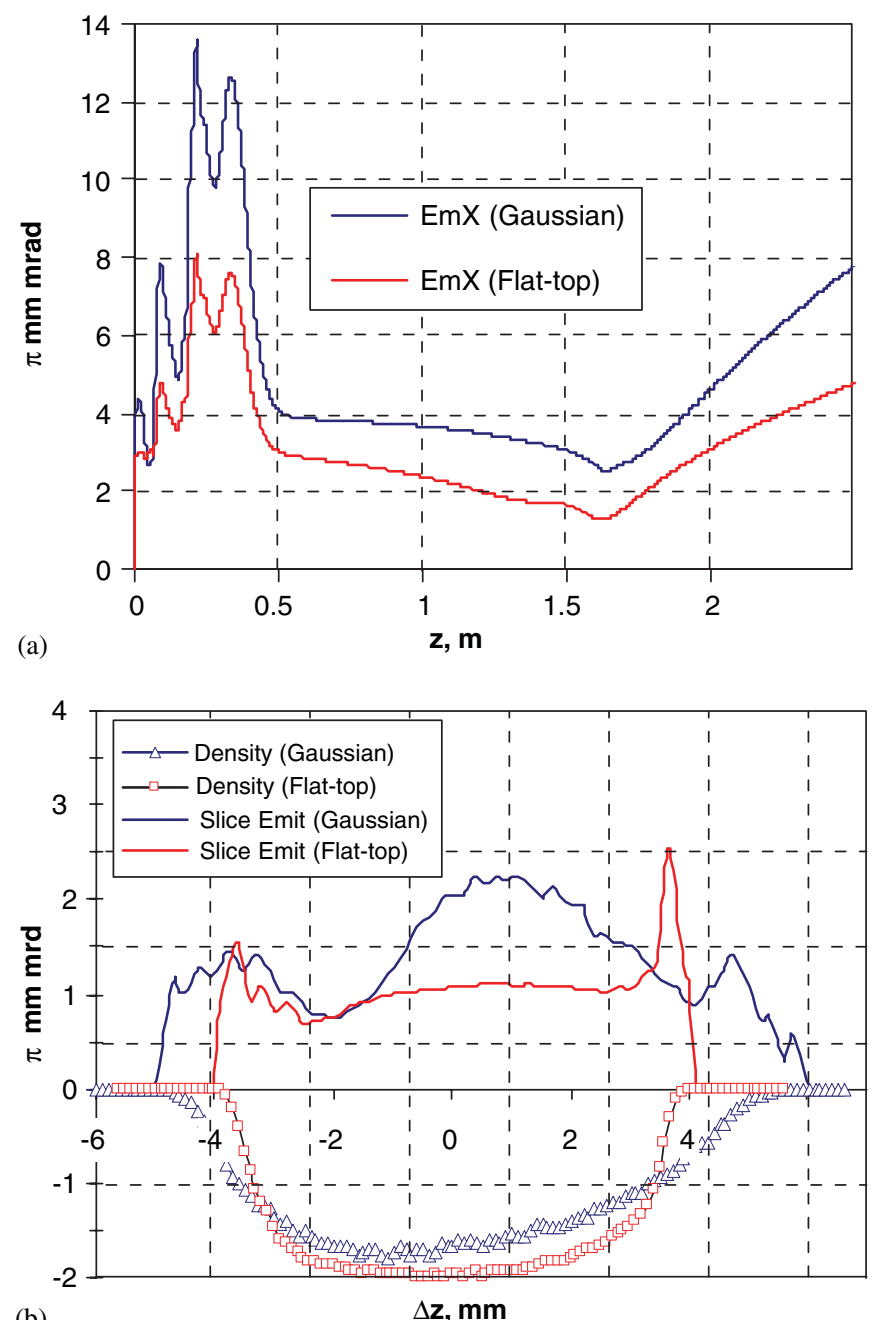

(b)

$\Delta \mathbf{z}, \mathbf{m m}$

Fig. 2. (a) Projected normalized beam emittance along the beam line for two longitudinal profiles: Gaussian $\left(\sigma_{\mathrm{t}} \approx 5.2 \mathrm{ps}\right.$, transverse profile: homogeneous, $\sigma_{x}=\sigma_{y}=0.5 \mathrm{~mm}$ ) and flat-top ( $20 \mathrm{ps}$ FWHM, $2 \mathrm{ps}$ rise/ fall time, transverse profile: homogeneous, $\sigma_{x}=\sigma_{y}=0.75 \mathrm{~mm}$ ). (b) Slice emittance for both longitudinal laser profiles at the EMSY position $(z=1.62 \mathrm{~m}$ from the photocathode). Charge densities are plotted in arbitrary units on the negative axis.

order to reduce the space charge influence on the transverse beam emittance the longitudinal laser profile at PITZ has been modified from a Gaussian $\left(\sigma_{\mathrm{t}} \approx 7 \mathrm{ps}\right)$ to a flat-top distribution (18-23 ps FWHM). ASTRA [3] beam dynamics simulations for both laser profiles show that the optimum emittance (minimum emittance at EMSY loca- tion $z=1.62 \mathrm{~m}$ ) for the flat-top profile (20 ps FWHM, 2 ps rise/fall time) is a factor of $\sim 2$ smaller than for the best Gaussian profile $\left(\sigma_{\mathrm{t}} \approx 5.2 \mathrm{ps}\right)$. The simulated normalized beam emittance as a function of longitudinal position along the beam line is shown in Fig. 2a. The slice beam emittance inside the electron bunch at $z=1.62 \mathrm{~m}$ is shown in Fig. $2 b$ for both longitudinal laser profiles together with the corresponding charge density profiles.

\section{Charge production study}

Faraday Cups and integrating current transformers (ICT) have been used to measure the electron beam charge. A basic measurement is the so-called phase scan: the accelerated charge downstream of the gun is measured as a function of the launch phase, the relative phase of the laser pulses with respect to the RF. Phase scan studies (measurements and simulations) for the normal operating
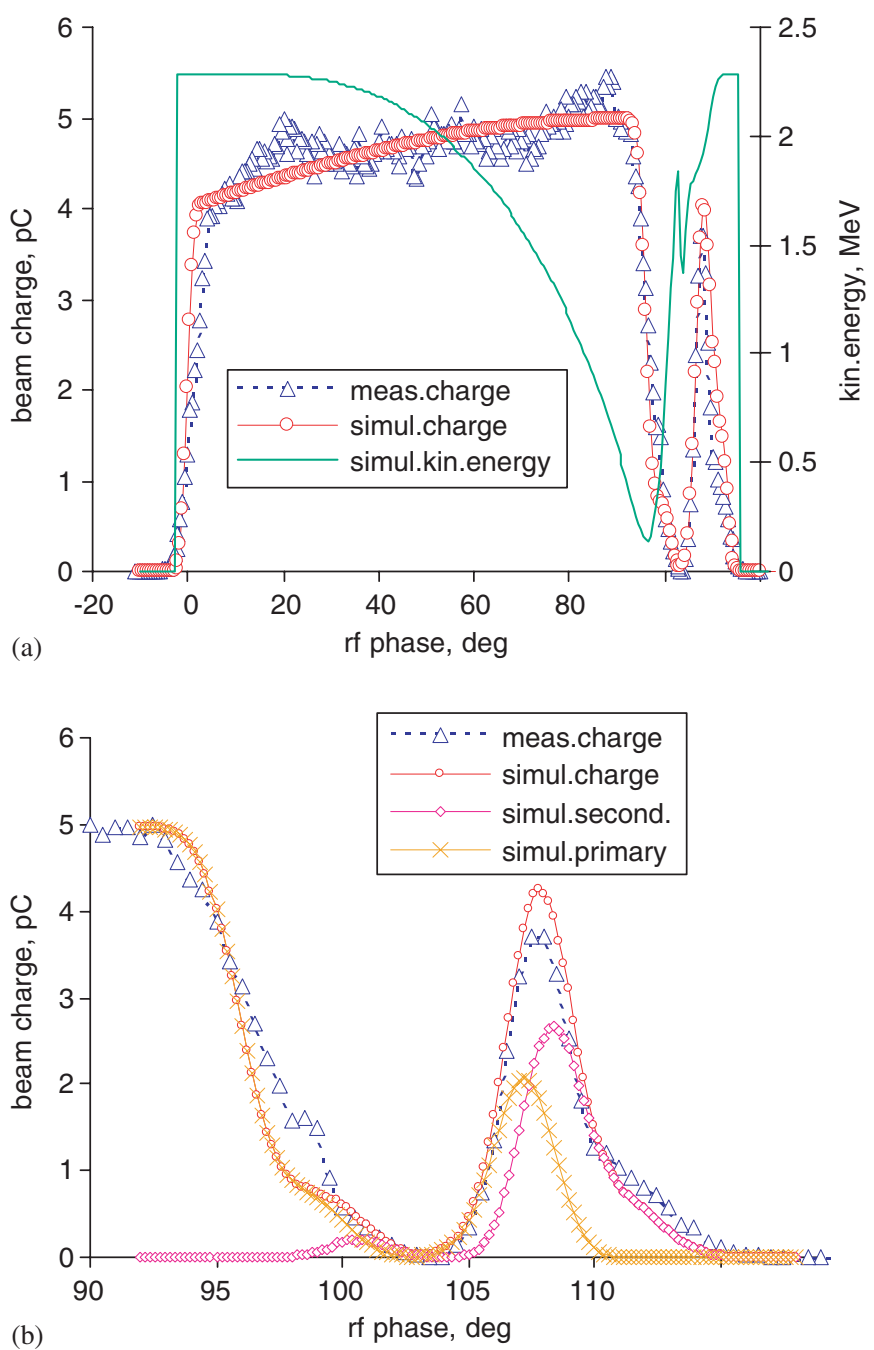

Fig. 3. (a) Measured and simulated phase scan (beam charge vs. RF phase). The simulated mean kinetic energy is plotted on the right axis. (b) Detailed phase scan for the "bump" phases. The simulated contributions of primary and secondary electrons (simulated) are shown in addition to their sum (simulated charge). 
conditions ( $1 \mathrm{nC}$ bunch charge and $42 \mathrm{MV} / \mathrm{m}$ accelerating field at the cathode) have been presented in Refs. [1,4]. For a detailed study of photo injection processes phase scans have been done also for low bunch charges $(\sim 4-5 \mathrm{pC})$ and moderate RF field on the photocathode $(\sim 21 \mathrm{MV} / \mathrm{m})$. Corresponding simulations have been performed as well. Solenoids were off for this study. A typical phase scan for this case is shown in Fig. 3. In order to simulate the phase scan the Schottky effect - the dependence of the charge emission on the actual electric field ( $R F$ and space charge) - has been modeled by using the formula $Q[\mathrm{pC}] \propto\left(0.4+0.045 \cdot E_{\mathrm{RF}+\mathrm{SC}}[\mathrm{MV} / \mathrm{m}]\right)$. Another charge production mechanism can be illustrated by a detailed study of the RF phase range $\sim 100-115^{\circ}$. This corresponds to a "bump" in the phase scan. For these RF phases the beam acceleration takes place in a non-smooth way: besides the particles accelerated downstream there is an amount of particles reflected back to the cathode. Under some circumstances reflected particles can produce secondary electrons. Simulations show (Fig. 3b) that secondary electrons can explain at least partially these phase scan peculiarities. Furthermore, momentum measurements and simulations [5] show that the beam momentum distribution contains a secondary electron part.

The discrepancy between measurements and simulations can be explained by a non-perfect beam and beamline alignment during measurements $\left(Q^{\text {measured }}<Q^{\text {simulated }}\right.$ due to electrons lost on the aperture), as well as by restrictions of the primary and secondary emission modeling $\left(Q^{\text {measured }}>Q^{\text {simulated }}\right)$. Another probable reason of the discrepancy is the uncertainty in the RF field balance of the gun cavity (ratio $E^{\text {Cathode }} / E^{\text {FullCell }}$ ) that has been used in the simulations.

\section{Emittance study at PITZ}

Measurements of the transverse emittance were performed using a single-slit scan technique [1]. Beamlets from three slit positions were taken into account for the emittance calculation. Beamlet profiles were observed at screen 3,1010 $\mathrm{mm}$ downstream of the single-slit mask (see Fig. 1).

Besides the photocathode laser parameter optimization other RF gun parameters have been optimized in order to reach a minimum normalized beam emittance, namely RF phase, main and bucking solenoid current. The emittance has been measured for different RF phases and main solenoid currents $\varepsilon_{x y}\left(\Phi_{\mathrm{RF}}, I_{\text {main }}\right)$ [6]. The photocathode laser with flat-top longitudinal profile ( $20 \mathrm{ps} \mathrm{FWHM} \mathrm{and}$ $\sim 5 \mathrm{ps}$ rise/fall time) and transverse $\mathrm{rms}(X / Y)$ sizes $0.51 /$ $0.63 \mathrm{~mm}$ has been tuned in order to keep the beam charge at $1 \mathrm{nC}$. The applied RF gradient on the cathode corresponded to $\sim 42 \mathrm{MV} / \mathrm{m}$. During the two-dimensional $\left(\Phi_{\mathrm{RF}}, I_{\text {main }}\right)$ parameter scan the bucking solenoid was off, so the magnetic field at the cathode is supposed to be small but not zero. For the best point $\left(\Phi_{\mathrm{RF}}=-5^{\circ}, I_{\text {main }}=\right.$ $305 \mathrm{~A})$ the bucking solenoid current was fine tuned in order to compensate the remnant longitudinal magnetic field on the cathode. The measured horizontal and vertical emittance as a function of the bucking solenoid current is shown in Fig. 4a. The asymmetry in the laser transverse profile leads to the observed beam shape and emittance asymmetry. Another asymmetry factor can be the impact of the vacuum mirror, used to position the laser beam onto the photocathode [7].

The normalized beam emittance has been simulated using ASTRA for injector parameters close to the ones observed during the emittance measurements, except the transverse laser profile which has been assumed rotationally symmetric (to apply a 2D $r-z$ space charge routine with equivalent rms sizes). The results of the simulations are shown in Fig. $4 \mathrm{~b}$. Simulated slice parameters of the optimum beam emittance $\left(I_{\text {buck }}=20 \mathrm{~A}\right)$ are plotted in Fig. 5a,b. for $z=1.62 \mathrm{~m}$ (EMSY location).
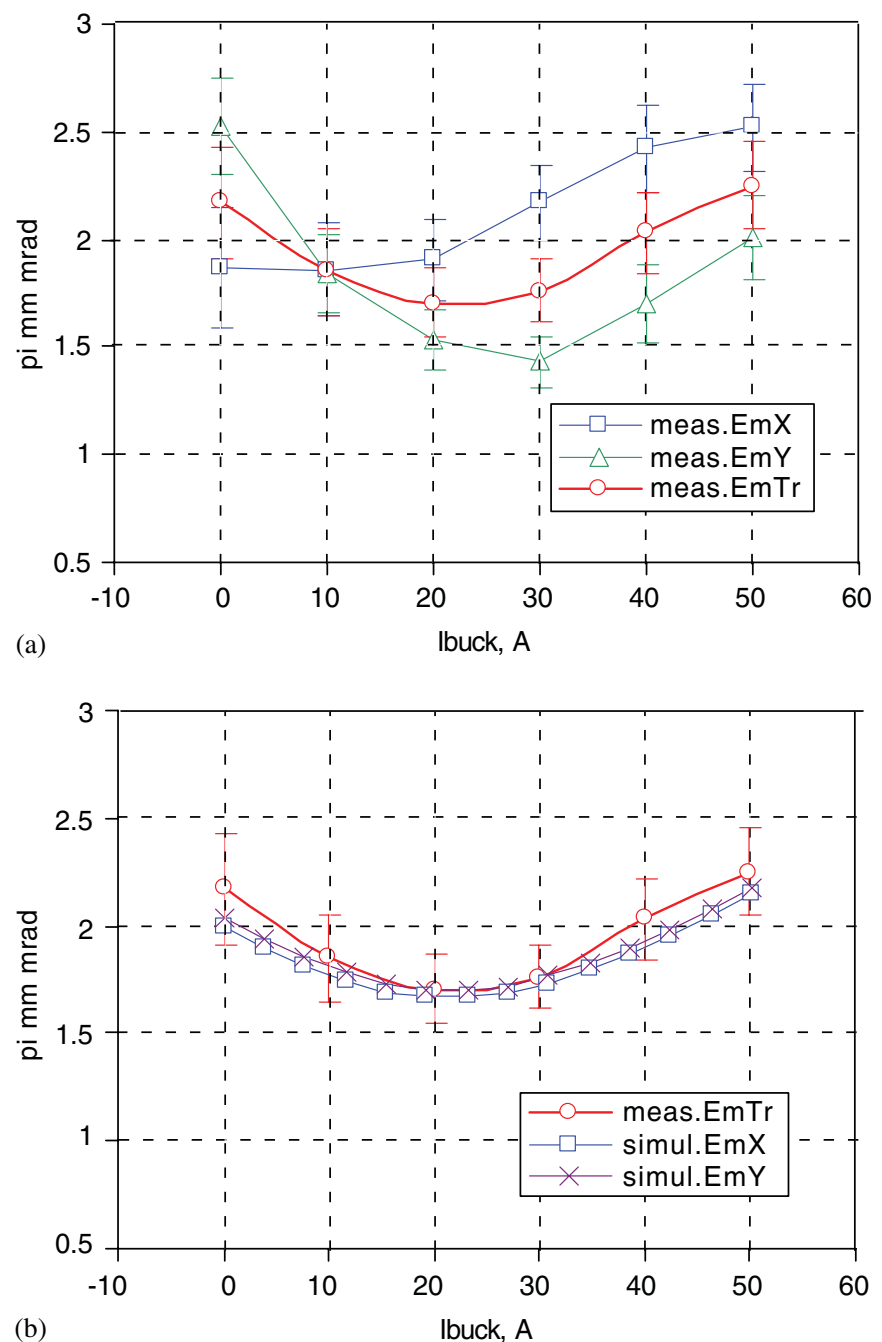

Fig. 4. (a) Measured normalized beam emittance as a function of the bucking solenoid current. (b) Measured transverse emittance $\left(\varepsilon_{\mathrm{tr}}=\right.$ $\sqrt{\varepsilon_{x} \cdot \varepsilon_{y}}$ ) and simulated horizontal and vertical emittance vs. bucking solenoid current. 


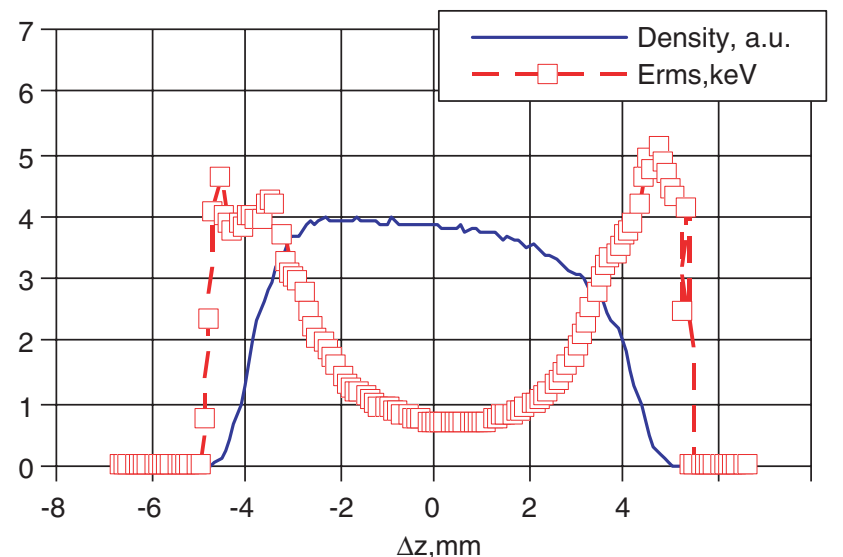

(a)

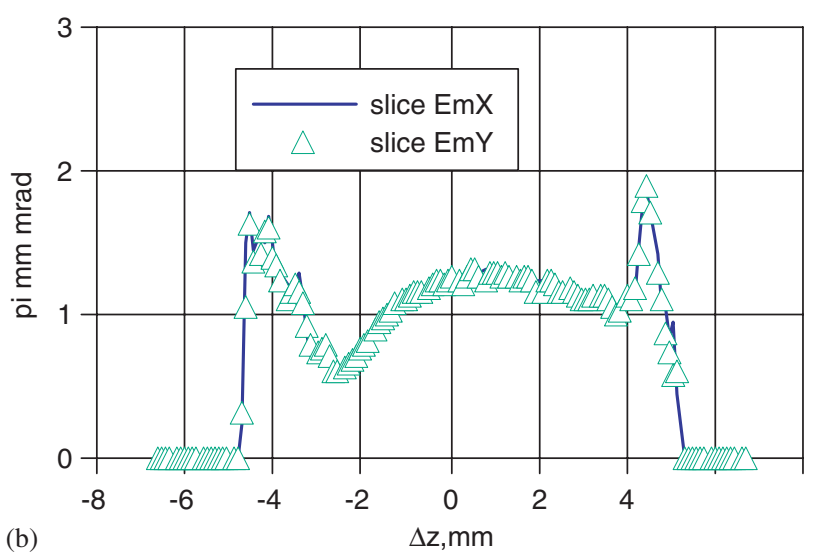

Fig. 5. Slice parameters for the optimum beam at $z=1.62 \mathrm{~m}$ : (a) Charge density and slice energy spread. (b) Slice horizontal and vertical emittance. The projected transverse normalized beam emittance is $1.7 \pi \mathrm{mm} \mathrm{mrad}$.

\section{Conclusions}

The optimization of the electron source at the Photo Injector Test facility at DESY Zeuthen (PITZ) yielded a minimum normalized projected emittance of $1.5 \pi \mathrm{mmmrad}$ in one plane and of $1.7 \pi \mathrm{mmmrad}$ as a geometrical average of the horizontal and vertical emittances. This result has been obtained by extensive variation of the photo injector parameters within a wide range. A flat-top longitudinal photocathode laser profile has been used. Detailed experimental and numerical studies of the photo injector have been performed. As next step, a large extension of the facility by including a booster cavity for emittance conservation studies is foreseen as well as further improvements of the photocathode laser properties.

\section{References}

[1] M. Krasilnikov, et al., Characterization of the electron source at the photo injector test facility at DESY Zeuthen, FEL 2003, Tsukuba, September 2003.

[2] SASE FEL at the TESLA Facility, Phase2, TESLA FEL Reports 2002-01.

[3] ASTRA User Manual, http://www.desy.de/ mpyflo/Astra_documentation/.

[4] M.v. Hartrott, et al., Experimental characterization of the electron source at the photo injector test facility at DESY Zeuthen, PAC 2003, Portland, May 2003.

[5] J.H. Han, M. Krasilnikov, K. Flöttmann, Secondary electron emission in a photocathode rf gun, Phys. Rev. ST Accel. Beams 8 (2005) 033501.

[6] M. Krasilnikov et al., Optimizing the PITZ electron source for the VUV-FE, in: Proceedings of the Ninth European Particle Accelerator Conference (EPAC2004), Lucerne, 2004, pp. 360-362.

[7] S. Setzer, et al., Inuence of beam tube obstacles on the emittance of the PITZ photoinjector, in: Proceedings of the Ninth European Particle Accelerator Conference (EPAC2004), Lucerne, 2004, pp. 1984-1986. 